\title{
ANÁLISE DA IMPERMEABILIZAÇÃO DOS SOLOS E AUMENTO DOS PI- COS DE VAZÃO DE CHEIAS EM BACIAS HIDROGRÁFICAS URBANAS
}

\author{
IMPERMEABILITY GRADE ANALYSIS OF SOILS AND INCREASE OF \\ MAXIMUM DISCHARGE IN URBAN HYDROGRAPHIC BASIN
}

\author{
Fabrizia Gioppo Nunes ${ }^{1}$ \\ Alberto Pio Fiori ${ }^{2}$
}

\begin{abstract}
RESUMO
Este trabalho trata da aplicação de um modelo hidrológico que transforma chuva em vazão, atrelado à utilização de técnicas de tratamento de dados em ambiente SIG, para quantificar e representar a distribuição espacial dos componentes hidrológicos de uma bacia hidrográfica de médio porte. O modelo hidrológico que tem por base a análise dos efeitos dos impactos da urbanização sobre o sistema de drenagem, utiliza a técnica de quantificação de áreas impermeáveis e da representação das características superficiais do terreno para simular picos de vazão de cheias, em bacias hidrográficas urbanas a parcialmente urbanizadas. Para a quantificação das áreas impermeáveis foram confeccionadas cartas temáticas da cobertura superficial do terreno através de técnicas de fotointerpretação digital. O aumento do escoamento superficial e do pico de vazão de cheias foram quantificados a partir da aplicação da fórmula racional, modificada e adaptada para bacia de médio porte. Para tais cálculos, foi necessário definir alguns parâmetros hidrológicos tais como intensidade da chuva, tempo de concentração, grupos hidrológicos de solos, coeficiente de retardo por armazenamento e o coeficiente de escoamento superficial do terreno. Todos os parâmetros considerados foram analisados em conjunto e integrados em ambiente SIG, tendo como resultado final, a quantificação dos coeficientes de escoamento superficial e a análise dos picos de vazão de cheias, dentro de uma visão multitemporal e de uma projeção futura. O procedimento metodológico desenvolvido provou ser uma boa alternativa para a distribuição espacial dos parâmetros hidrológicos e da estimativa de vazão de cheias em bacias hidrográficas de médio porte, que não possuam dados fluviométricos.
\end{abstract}

Palavras-chave: impermeabilização; urbanização; coeficiente de escoamento; vazão de cheias.

1 Doutora em Geologia pela Universidade Federal do Paraná. Professora substituta do Departamento de Geografia da Unversidade Federal do Paraná.

2 Pós-Doc pela Universidade de Siena, Itália. Professor Adjunto da Universidade Federal do Paraná. 
NUNES, F. G.; FIORI, A. P. Análise da impermeabilização dos solos e aumento dos picos de vazão...

\begin{abstract}
The main idea of this work is to apply one hydrological model to evaluate the relationship between rainfall and runoff of medium size basins. Data treatment and thematically maps made under GIS environment were used to quantify the spatial distribution of several hydrological components. The hydrological model created considers proper characteristics of the area under study and it was based on urbanization impact over drainage systems, where simulated peaks of discharge obtained quantify areas of impermeable surfaces. Cartographic bases of surface covering of land of 1962, 1980 and 2000 were accomplished by digital photogrammetry technique. For the digital photointerpretation, the software Erdas Imagine 8.6 ("Stereo Analyst" function) was run to obtain surface covering maps. The increases of runoff coefficient and maximum discharge were quantified using a modification of Rational Equation. In order to run these calculations, some hydrological parameters were established: rainfall intensity, basin physical characteristics including size, talvegue length, concentration time, soil hydrologic groups, soil antecedence humity, declivity land surface, effective precipitation, potential maximum retention ( $S$ ), time of hydrogram pick, reduction factor of hydrogram and runoff coefficient (C). During GIS step a data set containing thematic information of basin surface combining maps confectioned and tables of information by algebric operations were created. Simulations of maximum discharge were performed applying several thematic maps of soil, geology, land declivity, surface covering, potential maximum retention and runoff indices, all created by digital methods. All the parameters considered were analyzed together and integrated in GIS environmental considering a multi-temporal overview and a future projection. As a final result, by quantification and mapping for potential maximum retention of the soils were obtained, runoff coefficient and maximum discharge. The methodology developed here (based in digital photogrametria and GIS) seems to be an interesting alternative for analyzing spatial distribution of hydrologic parameters and estimate maximum discharge in hydrographic basins of medium size not containing fluviometric data.
\end{abstract}

Keywords: impermeability; urbanisation; runoff coefficients; maximum discharge.

\section{INTRODUÇÃO}

O presente trabalho apresenta uma adaptação do Método Racional para bacias urbanas a parcialmente urbanizadas de médio porte, através da representação da variabilidade espacial dos seus principais componentes hidrológicos.

O Método Racional, que é um modelo hidrológico de transformação de chuva em vazão, foi adaptado de modo a possibilitar uma melhor compreensão do comportamento hidrológico de uma bacia hidrográfica, podendo ser utilizado para quantificar e investigar as mudanças nos parâmetros hidrológicos, causadas pela alteração da cobertura superficial do solo, tais como:

a) mudanças no comportamento de infiltração e armazenamento da camada superficial do solo;

b) variação do tempo de concentração da bacia; c) mudanças no volume de escoamento superficial;

d) aumento das vazões de pico.

A adaptação do Método Racional foi feita a partir do aprimoramento dos procedimentos que determinam os parâmetros necessários à aplicação da fórmula racional. Em relação à fórmula que the serviu de base, o método foi modificado nos algoritmos que envolvem a representação dos processos de escoamento superficial do terreno, considerando os seguintes aspectos:

a) diversidade da cobertura superficial do terreno e a contribuição que cada parcela homogênea, corresponde dentro da área da bacia;

b) variabilidade espacial dos tipos hidrológicos do solo e da declividade do terreno;

c) retardamento do fluxo d'água por armazenamento na bacia, em função das declividades do terreno. 
d) umidade antecedente do solo;

e) determinação do tempo de concentração para bacias urbanas de médio porte;

f) variabilidade espacial da precipitação através do cálculo da precipitação média na bacia.

No método proposto, além da realização da cartografia dos principais parâmetros envolvidos, objetivou-se também a quantificação do aumento do escoamento superficial e da vazão de pico, dentro de uma análise multitemporal e de uma projeção futura.

Por ser de fácil aplicação e permitir o ajuste de seus parâmetros distribuídos espacialmente, a fórmula racional adaptada às necessidades de uma bacia de médio porte, possibilitou a comparação direta do aumento da vazão de pico com o aumento do coeficiente de escoamento superficial, gerados pela impermeabilização urbana.

\section{LOCALIZAÇÃO E CARACTERÍSTICAS DA ÁREA ANALISADA}

A aplicação da fórmula racional foi realizada na bacia hidrográfica do rio Atuba, que possui uma área de $127,43 \mathrm{~km}^{2}$, situada no Primeiro Planalto Paranaense entre as coordenadas de $25^{\circ} 17^{\prime} 00^{\prime \prime}$ e $25^{\circ} 30^{\prime} 00^{\prime \prime}$ de latitudes sul e; $49^{\circ} 17^{\prime} 00^{\prime \prime}$ e $49^{\circ} 10^{\prime} 00^{\prime \prime}$ de longitudes oeste.

Sendo um dos principais afluentes da margem direita das nascentes do rio Iguaçu, a área drenada pelo rio Atuba engloba, além da porção nordeste de Curitiba, partes dos municípios de Pinhais, Colombo, Piraquara, São José dos Pinhais e Almirante Tamandaré (Figura 1).
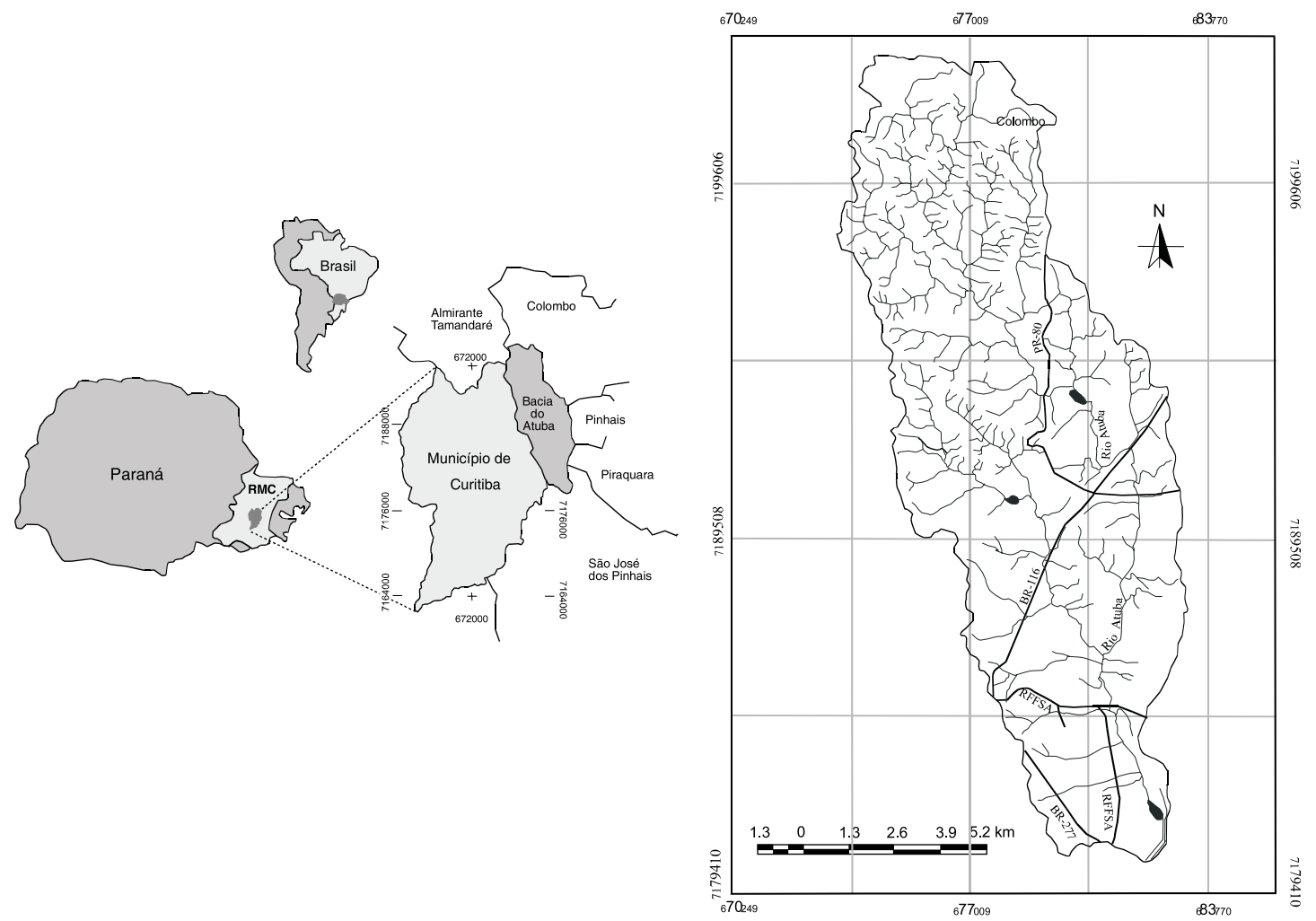

FIGURA 1 - LOCALIZAÇÃO DA ÁREA DE ESTUDO. LOCATION OF THE STUDIED AREA IN THE PARANÁ STATE 
NUNES, F. G.; FIORI, A. P. Análise da impermeabilização dos solos e aumento dos picos de vazão...

Por apresentar diversos registros de inundações em seu interior e áreas urbanas sujeitas a este tipo de impacto, a bacia hidrográfica do rio Atuba foi selecionada como área de estudo, para a aplicação do Método Racional. Outro fator importante que foi levado em consideração, durante a sua seleção, é que a bacia do rio Atuba vem sofrendo um grande processo de urbanização no decorrer dos últimos trinta anos, sendo considerada como a segunda bacia mais urbanizada de Curitiba e suas imediações.

Como principal característica geomorfológica temse a presença das maiores declividades em suas cabeceiras, onde se verificam os afluentes de pequenas extensões. As menores declividades encontram-se na região do baixo curso da bacia, sobre os terrenos encharcados de solos hidromórficos, com córregos de longas extensões.

Além dos solos hidromórficos, outras classes de solos são encontradas na bacia, em seu alto e médio curso como Latossolo, Cambissolos, Terra Roxa Estruturada e Solos Orgânicos.

\section{MATERIAIS E MÉTODOS}

O modelo hidrológico elaborado a partir do aperfeiçoamento da fórmula racional, para bacias de médio porte, teve por base o estudo dos impactos da urbanização sobre o sistema de drenagem, utilizandose da técnica de quantificação de áreas impermeáveis e da representação das características superficiais do terreno. Esta técnica serviu para quantificar e avaliar o escoamento superficial e simular os picos de vazão de cheias.

Para a quantificação das áreas permeáveis e impermeáveis, ao longo dos anos, realizou-se a análise multitemporal dos aspectos relacionados à evolução da cobertura superficial do terreno e redução das áreas naturais.

A análise multitemporal foi executada a partir da confecção de cartas temáticas da cobertura superficial do terreno dos anos de 1962, 1980 e 2000 com o emprego da técnica de fotointerpretação digital, através da função "Stereo Analyst" do software Erdas
Imagine $8.6^{\circledR}$. Como materiais de apoio foram utilizadas fotografias aéreas dos referidos anos, além de cartas topográficas digitalizadas e georreferenciadas, da Coordenação da Região Metropolitana de Curitiba (COMEC).

As classes temáticas de cobertura superficial do terreno, determinadas conforme o adensamento urbano e o tipo de uso e ocupação do solo foram:

- Edificação muito densa: partes centrais densamente construídas de uma cidade com cerca de $85 \%$ de impermeabilização;

- Edificação não muito densa: zonas residenciais adjacentes ao centro, de menor densidade de habitação que as regiões centrais, com cerca de 65\% de impermeabilização;

- Edificações com superfícies livres: zonas residenciais com cerca de $38 \%$ de impermeabilização;

- Edificações com muitas superficies livres: zonas residenciais com construções esparsas com cerca de $30 \%$ de impermeabilização;

- Zonas industriais e comerciais: áreas industrias e barracões de comércios com cerca de $72 \%$ de impermeabilização;

- Campo e áreas verdes: parques, praças, campos de esportes, áreas cultivadas, áreas com gramados e terrenos desnudos;

- Zonas florestais e vegetação densa: área com vegetação de porte arboreo natural ou reflorestadas em boas condições;

- Corpos d'água: lagos artificiais ou naturais.

A quantificação das classes da cobertura superficial do terreno, ao longo dos três anos considerados, tornou-se possível através da operação de análise espacial, realizada pelo Sistema de Processamento de Informações Georreferenciadas - Spring. Para tal operação foi executado o cálculo de áreas e verificada a percentagem de cada polígono, que representam nas cartas temáticas de 1962, 1980 e 2000 as classes de cobertura superficial do terreno (Tabela 1). 
NUNES, F. G.; FIORI, A. P. Análise da impermeabilização dos solos e aumento dos picos de vazão...

TABELA 1 - CÁLCULO DAS ÁREAS DAS CLASSES DE COBERTURA SUPERFICIAL DO TERRENO DA BACIA HIDROGRÁFICA DO RIO ATUBA DOS ANOS (1962, 1980 E 2000). CALCULATION OF AREAS FOR TYPES OF LAND COVER IN 1962, 1980, 2000

\begin{tabular}{|c|c|c|c|c|c|c|c|c|c|c|c|c|c|}
\hline \multirow{4}{*}{\multicolumn{2}{|c|}{ Descrição das Classes }} & \multicolumn{12}{|c|}{ Área na bacia do Atuba /Anos } \\
\hline & & \multicolumn{4}{|c|}{1962} & \multicolumn{4}{|c|}{1980} & \multicolumn{4}{|c|}{2000} \\
\hline & & \multirow{2}{*}{$\mathrm{Km}^{2}$} & \multirow{2}{*}{$\%$} & \multicolumn{2}{|c|}{$\begin{array}{l}\text { Total da } \\
\text { Classe }\end{array}$} & \multirow{2}{*}{$\mathrm{km}^{2}$} & \multirow{2}{*}{$\%$} & \multicolumn{2}{|c|}{$\begin{array}{l}\text { Total da } \\
\text { Classe }\end{array}$} & \multirow{2}{*}{$\mathrm{Km}^{2}$} & \multirow{2}{*}{$\%$} & \multicolumn{2}{|c|}{$\begin{array}{l}\text { Total da } \\
\text { Classe }\end{array}$} \\
\hline & & & & $\mathrm{km}^{2}$ & $\%$ & & & $\mathrm{~km}^{2}$ & $\%$ & & & $\mathbf{k m}^{2}$ & $\%$ \\
\hline \multirow{2}{*}{$\begin{array}{l}\text { Edificação } \\
\text { muito densa. }\end{array}$} & Permeável & 0,0335 & 0,03 & \multirow[b]{2}{*}{0,2032} & \multirow[b]{2}{*}{0,16} & 0,4760 & 0,37 & \multirow[b]{2}{*}{3,1734} & \multirow[b]{2}{*}{2,5} & 1,4343 & 1,12 & \multirow[b]{2}{*}{9,5618} & \multirow[b]{2}{*}{7,5} \\
\hline & Impermeável & 0,1697 & 0,13 & & & 2,6974 & 2,12 & & & 8,1275 & 6,38 & & \\
\hline \multirow{2}{*}{$\begin{array}{l}\text { Edificação } \\
\text { não muito } \\
\text { densa. }\end{array}$} & Permeável & 0,0000 & 0,0 & \multirow[b]{2}{*}{0,0000} & \multirow[b]{2}{*}{0,0} & 0,9797 & 1,43 & \multirow[b]{2}{*}{2,7991} & \multirow[b]{2}{*}{2,2} & 6,513 & 5,11 & \multirow[b]{2}{*}{18,607} & \multirow[b]{2}{*}{14,6} \\
\hline & Impermeável & 0,0000 & 0,0 & & & 1,8194 & 0,77 & & & 12,094 & 9,49 & & \\
\hline \multirow{2}{*}{$\begin{array}{l}\text { Edificações } \\
\text { com } \\
\text { superfícies } \\
\text { livres. }\end{array}$} & Permeável & 0,1644 & 0,13 & \multirow[b]{2}{*}{0,2651} & \multirow[b]{2}{*}{0,21} & 5,5838 & 4,38 & \multirow[b]{2}{*}{9,0061} & \multirow[b]{2}{*}{7,0} & 11,029 & 8,70 & & \\
\hline & Impermeável & 0,1007 & 0,1 & & & 3,4223 & 2,62 & & & 6,7598 & 5,30 & 17,789 & 14 \\
\hline Edificação & Permeável & 11,028 & 8,59 & & & 18,911 & 14,8 & & & 10,675 & 8,31 & & \\
\hline $\begin{array}{l}\text { superfícies } \\
\text { livres. }\end{array}$ & Impermeável & 4,7265 & 3,75 & 15,755 & 12,3 & 8,1048 & 6,36 & 27,016 & 21,2 & 4,5750 & 3,59 & 15,250 & 11,9 \\
\hline Zonas & Permeável & 0,0672 & 0,05 & & & 1,3113 & 1,04 & & & 2,3044 & 1,75 & & \\
\hline comerciais. & Impermeável & 0,1728 & 0,15 & 0,2400 & 0,2 & 3,3719 & 2,66 & 4,6833 & 3,7 & 5,9257 & 4,65 & 8,2301 & 6,4 \\
\hline $\begin{array}{c}\text { Campo e } \\
\text { areas verdes. }\end{array}$ & Permeável & 76,543 & 60,1 & 76,543 & 60,1 & 53,638 & 42,1 & 53,638 & 42,1 & 27,109 & 21,2 & 27,109 & 21,2 \\
\hline $\begin{array}{l}\text { Zonas } \\
\text { florestais e } \\
\text { vegetação } \\
\text { densa. }\end{array}$ & Permeável & 34,367 & 26,9 & 34,367 & 26,9 & 27,050 & 21,2 & 27,050 & 21,2 & 30,747 & 24,1 & 30,747 & 24,1 \\
\hline $\begin{array}{l}\text { Corpos } \\
\text { d'água. }\end{array}$ & & & & & 0,05 & & 0,0605 & & 0,05 & & & 1397 & 0,11 \\
\hline Área total das & Permeável & 122,20 & 95,6 & & & 107,95 & 85,3 & & & 89,812 & 70,0 & & \\
\hline classes. & Impermeável & 5,1697 & 4,5 & 127,43 & 100 & 19,416 & 14,5 & 127,43 & 100 & 37,48 & 30,0 & 127,43 & 100 \\
\hline
\end{tabular}

O aumento do escoamento superficial do terreno e aumento dos picos de vazão de cheias foram calculados pela aplicação da fórmula racional, exigindo a definição de alguns parâmetros como intensidade da chuva, características físicas da bacia (área, comprimento do talvegue, tempo de concentração, grupos hidrológicos de solos, umidade antecedente dos solos, declividade do terreno entre outros), coeficiente de armazenamento por infiltração, coeficiente de retardo por efeito da declividade e o coeficiente de escoamento superficial do terreno.

Na etapa de SIG, foi criado um banco de dados contendo as informações temáticas da superfície da bacia, através da confecção e combinação de cartas e tabelas por operações algébricas.

Para a simulação da vazão de pico foram utilizadas diversas cartas temáticas elaboradas em meio digital como carta de solos, carta geológica, carta de declividade do terreno, cartas de coberturas superficiais do terreno, cartas de índices de armazenamento (coeficiente S) e cartas de índices de escoamento superficial do terreno (coeficiente $\mathrm{C}$ ), além de outras informações que fazem parte dos parâmetros necessários à aplicação do método proposto.

Todos os parâmetros considerados foram analisados em conjunto e integrados em ambiente SIG visando, como resultado final, a quantificação do escoamento superficial e a análise dos picos de vazão de cheias, dentro de uma visão multitemporal.

O percentual de áreas impermeáveis e do coeficiente "C", para o cenário futuro (ano de 2020) foi estimado através da confecção gráfica dos dados existentes dos anos de 1962, 1980 e 2000, com a projeção da inflexão da curva de tendência desses dados, para o ano de 2020. 
NUNES, F. G.; FIORI, A. P. Análise da impermeabilização dos solos e aumento dos picos de vazão...

\section{MÉTODO RACIONAL}

O Método Racional é bastante utilizado para o estudo de picos de cheias em pequenas bacias hidrográficas, sendo a vazão de pico determinada em função da precipitação e das características do recobrimento da bacia, tendo como base principal o emprego da fórmula racional (Fendrich et al. 1997).

No trabalho os cálculos das vazões de cheias foram realizados pela fórmula racional, modificada e adaptada, através da inclusão do coeficiente de retardo que reflete o efeito de armazenamento d'água na bacia, devido às diferenças da declividade do terreno, ou seja:

$$
Q_{p}=\frac{C r_{m} \cdot i_{m} \cdot A}{3,6} \quad \text { equação (1) }
$$

$Q_{p}=$ vazão de pico, em $\mathrm{m}^{3} / \mathrm{s}$;

$\mathrm{Cr}_{\mathrm{m}}=$ coeficiente de escoamento superficial real médio da bacia;

$\mathrm{i}_{\mathrm{m}}=$ intensidade máxima da chuva sobre toda a área drenada, para tempo de duração igual ao tempo de concentração da bacia, em $\mathrm{mm} / \mathrm{h}$;

$\mathrm{A}=$ área da bacia em $\mathrm{km}^{2}$.

Partindo do princípio que as limitações do Método Racional, usado na sua forma original, podem implicar em erros grosseiros quando aplicado em bacias superiores a 0,50 $\mathrm{km}^{2}$ Fendrich et al. (1997), durante o trabalho procurou-se, calcular todos os seus parâmetros de modo a representarem o melhor possível a heterogeneidade e variações espaciais dos elementos fisiográficos da bacia.

Estas considerações ajudaram a minimizar a generalização das características dos elementos físicos da paisagem para toda a área da bacia, uma vez que este efeito não é contemplado no Método Racional, em sua concepção original.

\section{TEMPO DE CONCENTRAÇÃO $\left(t_{c}\right)$}

No Método Racional a duração da chuva crítica, ou seja, a duração da chuva que irá ocasionar a vazão de pico é igual ao tempo de concentração desta na área da bacia. Sendo o tempo de concentração definido como o intervalo de tempo, contado a partir do início da chuva, para que toda a bacia esteja contribuindo na seção de saída.

$\mathrm{Na}$ literatura técnico-científica são diversas as fórmulas apresentadas para o cálculo do tempo de concentração, tanto para áreas rurais como para áreas urbanas. A maioria destas fórmulas, principalmente as de aplicação em áreas urbanas, é elaborada a partir de observações de bacias de micro escalas, podendo ser usadas apenas nestas áreas.

Partindo das constatações expostas acima, o tempo de concentração na bacia hidrográfica do rio Atuba foi calculado pela equação de Germano et al. (1998), que permite a determinação deste parâmetro para bacias urbanas de médio porte.

Os autores (Germano et al. 1998) estudaram o comportamento hidrológico de 28 bacias brasileiras urbanas de médio porte, distribuídas nas cidades de Porto Alegre, Joinville, São Paulo, Rio de Janeiro e Curitiba, estabelecendo correlações entre o comprimento destas bacias e a percentagem de suas áreas impermeáveis. Obtiveram a seguinte expressão:

$$
\text { tc }=18,628 \frac{L t a I^{0,882}}{I M P^{0,272}} \text { equação (2) }
$$

onde:

$\mathrm{t}_{\mathrm{c}}=$ tempo de concentração em minutos;

Ltal = extensão do talvegue em quilômetros;

IMP = percentagem de áreas impermeáveis na bacia.

Para possibilitar uma análise multitemporal do processo de impermeabilização do solo, o tempo de concentração $\left(t_{c}\right)$ na bacia hidrográfica do rio Atuba foi determinado para quatro períodos distintos de desenvolvimento urbano, sendo eles:

a) início da década de 60 (ano de 1962) quando a impermeabilização do solo ocupava apenas $4,5 \%$ da área total da bacia, estando esta quase que em seu estado natural;

b) década de 80 (1980), início do processo de urbanização na bacia e intensificação da impermeabilização;

c) ano 2000, período com nível de urbanização e impermeabilização do solo, próximo ao atual;

d) cenário futuro, equivalente a uma projeção correspondente a um estágio de impermeabilização do solo para o ano de 2020. 
NUNES, F. G.; FIORI, A. P. Análise da impermeabilização dos solos e aumento dos picos de vazão...

A escolha das datas para o cálculo do tempo de concentração ocorreu em função dos dados de fotografias aéreas disponíveis para o mapeamento da cobertura superficial do terreno. Na Tabela 2 são apresentados os valores do tempo de concentração da bacia do rio Atuba, calculados para os quatro períodos de desenvolvimento urbano determinados.

TABELA 2 - VALORES DO TEMPO DE CONCENTRAÇÃO DA BACIA HIDROGRÁFICA DO RIO ATUBA. TIME OF CONCENTRATION VALUES FOR ATUBA RIVER HYDROGRAPHIC BASIN

\begin{tabular}{|c|c|c|c|c|c|c|}
\hline Anos & A (área da bacia) & Ltal (extensão do talvegue) & $\mathbf{A}_{\mathbf{i}} \mathbf{k m}^{2}$ & $\mathbf{A}_{\mathbf{i}} \%$ & $\mathbf{t}_{\mathrm{c}} \mathbf{m i n}$ & $\mathbf{t}_{\mathrm{c}} \mathbf{h}$ \\
\hline 1962 & 127,43 & $23,50 \mathrm{~km}$ & 5,20 & 4,5 & 199,73 & 3,35 \\
\hline 1980 & 127,43 & $23,50 \mathrm{~km}$ & 18,48 & 14,5 & 145,70 & 2,43 \\
\hline 2000 & 127,43 & $23,50 \mathrm{~km}$ & 37,71 & 30,0 & 119,68 & 2,00 \\
\hline 2020 & 127,43 & $23,50 \mathrm{~km}$ & 57,98 & 45,5 & 106,95 & 1,45 \\
\hline
\end{tabular}

\section{INTENSIDADE MÁXIMA DA CHUVA $\left(i_{m}\right)$}

A intensidade máxima da chuva utilizada no cálculo das vazões de pico foi determinada pela equação de chuvas intensas, proposta por Fendrich (2000).

A equação de chuvas intensas foi elaborada através da relação de intensidade-duração-frequência de registros pluviométricos observados na Estação Pluviométrica de Curitiba - Prado Velho entre os anos de 1981 a 1999. Utilizando-se de ajustes estatísticos Fendrich (2000), determinou os valores de a, b, c e d para a região de Curitiba, obtendo a seguinte equação:

$$
i_{m}=\frac{5.726,64 . T_{r}^{0,159}}{\left(t_{d}+41\right)^{1,041}} \quad \text { equação (3) }
$$

onde:

$\mathrm{i}_{\mathrm{m}}=$ intensidade da chuva dada em $\mathrm{mm} / \mathrm{h}$;

$\mathrm{T}_{\mathrm{r}}=$ tempo de retorno em anos;

$t_{d}=$ duração da chuva, em min que é igual ao tempo de concentração da bacia.

Durante o trabalho foram selecionados quatro períodos de retorno diferentes para a aplicação da equação de chuvas intensas. A Tabela 3 apresenta os valores da intensidade da chuva obtidos para os tempos de concentração determinados e respectivos anos analisados.

TABELA 3 - VALORES DA INTENSIDADE DA CHUVA PARA A BACIA HIDROGRÁFICA DO RIO ATUBA. INTENSITY OF RAIN VALUES FOR ATUBA RIVER HYDROGRAPHIC BASIN

\begin{tabular}{|c|c|c|c|c|c|}
\hline \multirow{2}{*}{ Ano } & \multirow{2}{*}{$\begin{array}{c}\text { Tempo de } \\
\text { concentração }\end{array}$} & \multicolumn{4}{|c|}{ Intensidade da chuva $(\mathrm{mm} / \mathrm{h})$} \\
\cline { 3 - 6 } & $\left(\mathrm{t}_{\mathrm{c}}\right) \mathrm{min}$. & 5 & 10 & 25 & 51,70 \\
\cline { 3 - 6 } & 199,73 & 24,55 & 27,40 & 41,30 & 35,40 \\
\hline 1962 & 145,70 & 31,97 & 35,70 & 48,28 & 46,10 \\
\hline 1980 & 119,68 & 37,40 & 41,75 & 52,60 & 5,90 \\
\hline 2000 & 106,95 & 40,75 & 45,48 & 58,75 \\
\hline 2020 & & &
\end{tabular}

\section{COEFICIENTE DE ESCOAMENTO SUPERFICIAL REAL MÉDIO DA BACIA $\left(\mathrm{Cr}_{\mathrm{m}}\right)$}

A estimativa do coeficiente de escoamento superficial na bacia hidrográfica do rio Atuba foi feita pelo emprego do Método do Soil Conservation Service (SCS), elaborado pelo Departamento de Agricultura dos Estados Unidos.

A metodologia do SCS é fundamentada em um parâmetro que busca descrever o tipo de uso do solo e a condição da superfície do terreno, em relação ao potencial de gerar escoamento superficial. Este parâmetro é representado pela sigla CN (curva número).

O valor de CN é compreendido entre zero e 100, sendo zero a representação de uma bacia de condutividade hidráulica infinita e 100 o valor correspondente a uma bacia totalmente impermeável.

Segundo Chow et al. (1988) o algoritmo do SCS está baseado na proporção: 
NUNES, F. G.; FIORI, A. P. Análise da impermeabilização dos solos e aumento dos picos de vazão...

$$
\frac{(P-C)}{S}=\frac{C}{P} \quad \text { equação (4) }
$$

onde:

$\mathrm{P}=$ precipitação acumulada, ou seja, a precipitação total de um evento considerado $(\mathrm{mm})$;

$C=$ coeficiente de escoamento superficial (adimensional);

$S=$ coeficiente de armazenamento superficial, por infiltração, que está relacionada com os parâmetros que caracterizam a superfície (CN), dado em (mm).

A perda inicial de água que é igual à quantidade de precipitação que não produz escoamento no início da chuva de tormenta é considerado como $20 \%$ das perdas potenciais máximas, sendo:

$$
l a=0,2 * S \quad \text { equação (5) }
$$

Introduzindo a perda inicial (la) da precipitação acumulada $(P)$ na Equação 4 e isolando $(C)$ tem-se:

$$
C=\frac{(P-l a)^{2}}{(P-0,8 . S)} \quad \text { equação (6) }
$$

Por fim, substituindo a Equação (5) em (6), obtêm-se a seguinte relação:

$$
\begin{gathered}
C=\left[\frac{(P-0,2 . S)^{2}}{(P+0,8 . S)}\right] \cdot \frac{1}{P} \quad \text { para } P>l a \\
\quad \text { equação }(7)
\end{gathered}
$$

Quando P < la o valor de "C" será igual a zero

Para determinar a capacidade máxima de infiltração da camada superficial do solo (valor de "S"), o método do SCS relaciona esse parâmetro com o fator de CN pela seguinte expressão:

$$
S=\frac{25400}{C N}-254 \text { equação (8) }
$$

\section{DETERMINAÇÃO DOS VALORES DE (CN)}

Os valores de $\mathrm{CN}$ foram estimados a partir da utilização do modulo cálculo do "CN", para bacias urbanas a parcialmente urbanizadas, contido no modelo hidrológico-hidrodinâmico IPHS1 para Windons?, Versão 2.1, elaborado pelo Instituto de Pesquisas Hidráulicas da UFRGS e Faculdade de Engenharia Agrícola - UFPel.

O modelo fornece o valor de $\mathrm{CN}$ de acordo com a umidade antecedente dos solos a partir da interpolação das características da cobertura superficial do terreno, com cada grupo hidrológico de solos, considerado no método do SCS.

Durante o cálculo dos valores de CN foi considerada a condição III de umidade antecedente do solo, ou seja, solos úmidos próximos à saturação, onde as chuvas dos últimos 5 dias anteriores à tormenta analisada foram superiores a $40 \mathrm{~mm}$. Os grupos hidrológicos de solos determinados na bacia são:

Grupo C - solos argilosos com teor total de argila de $20 \%$ a $30 \%$, mas sem camadas argilosas impermeáveis ou contendo pedras, até a profundidade de 1,2 $\mathrm{m}$. Possuem baixa taxa de infiltração quando completamente úmidos. Este grupo de solos, na bacia do rio Atuba, é constituído pelos Latossolos Vermelho e Amarelo, Cambissolos e Terra Roxa Estruturada.

Grupo D - solos argilosos, com $30 \%$ a $40 \%$ de argila total, com camada densificada a uns $50 \mathrm{~cm}$ de profundidade e quase impermeável. São solos com elevado potencial de escoamento e baixa taxa de infiltração. Na bacia do rio Atuba este grupo de solos é constituído pelos Solos Hidromórfico e Solos Orgânicos.

Após a determinação dos valores de $\mathrm{CN}$ puderam ser calculados os valores de "S" pela equação (8) e destes, os valores de "C" pela equação (7). Os valores de $C N$, "S" e "C" foram determinados para cada tipo de cobertura superficial e grupo hidrológico de solos, considerando sempre uma perda inicial de $20 \%$.

Os valores de "C" utilizados durante a simulação dos picos de vazão de cheias foram calculados para a maior tormenta observada nos registros históricos das estações pluviométricas circunvizinhas à bacia, somada à chuva total dos cinco dias anteriores a esta tormenta. Desta precipitação foi extraída a média sobre toda a área da bacia, conforme a ponderação de observações pontuais a partir do Método do Polígono de Thiessen, obtendo-se um valor final de $176,96 \mathrm{~mm}$. 
NUNES, F. G.; FIORI, A. P. Análise da impermeabilização dos solos e aumento dos picos de vazão...

\section{DETERMINAÇÃO DOS COEFICIENTES DE RE- TARDO POR EFEITO DA DECLIVIDADE $(\varphi)$}

Conforme Souza Pinto et al. (1976), o coeficiente de retardo, que reflete o armazenamento de água na bacia hidrográfica, em função da declividade do terreno, pode ser determinado por:

$$
\varphi=\frac{1}{\sqrt[n]{10 . L t a l}} \quad \text { equação (9) }
$$

onde:

$\varphi=$ coeficiente de retardo, que reflete os efeitos de armazenamento da declividade;

Ltal = extensão do talvegue, em km;

$n=$ coeficiente em função da declividade da bacia.

Como resultado da aplicação da Equação 9, tem-se a Tabela 4, contendo os valores de " $n$ " fornecidos por Souza Pinto et al. (1976) e os valores de " $\varphi$ " calculados para a bacia do rio Atuba, em função da declividade do terreno.

TABELA 4 - VALORES DE "N" E " $\varphi$ " PARAA BACIA HIDROGRÁFICA DO RIO ATUBA. VALUES OF "N" AND " $\varphi$ " FOR ATUBA RIVER HYDROGRAPHIC BASIN

\begin{tabular}{|c|c|c|c|c|}
\hline Tipo de declividade & $\%$ & Valor de “ $n$ " & Ltal (km) & Valor de “ $\varphi$ ” \\
\hline declividade muito forte & $>25 \%$ & 3,5 & \multirow{2}{*}{0,25} \\
\hline declividade forte & 25 a $15 \%$ & 3,5 & 0,25 \\
\hline declividade média & 15 a $10 \%$ & 3 & 0,18 \\
\hline declividade fraca & $<10 \%$ & 2,5 & 0,12 \\
\hline
\end{tabular}

Os valores de " $\varphi$ " calculados foram embutidos nos coeficientes "C", obtendo-se novos valores denominados como coeficiente de escoamento superficial real "Cr" (Tabela 5).

TABELA 5 - VALORES DE "CR" PARA A TORMENTA SEVERA SELECIONADA E DURAÇÃO DE 6 DIAS. RUNOFF "CR" VALUES FOR MAXIMUM RAIN SELECTED WHICH LASTED 6 DAYS

\begin{tabular}{|c|c|c|c|c|c|c|}
\hline \multirow{3}{*}{ Tipo de Cobertura Superficial do Terreno } & \multicolumn{6}{|c|}{ Valores de "Cr" para condição-III de umidade antecedente do solo } \\
\hline & \multicolumn{2}{|c|}{$\begin{array}{l}\text { declividade forte } \\
\text { a muito forte }\end{array}$} & \multicolumn{2}{|c|}{ declividade média } & \multicolumn{2}{|c|}{ declividade fraca } \\
\hline & $\mathrm{C}$ & $\mathrm{D}$ & $\mathrm{C}$ & $\mathrm{D}$ & $\mathrm{C}$ & $\mathrm{D}$ \\
\hline $\begin{array}{l}\text { Edificação muito densa: em média 85\% de áreas } \\
\text { impermeáveis. }\end{array}$ & 0,24 & 0,24 & 0,17 & 0,17 & 0,12 & 0,12 \\
\hline $\begin{array}{l}\text { Edificação não muito densa: em média 65\% de áreas } \\
\text { impermeáveis. }\end{array}$ & 0,23 & 0,24 & 0,16 & 0,17 & 0,11 & 0,12 \\
\hline $\begin{array}{l}\text { Edificações com superfícies livres: em média 38\% de áreas } \\
\text { impermeáveis. }\end{array}$ & 0,22 & 0,22 & 0,16 & 0,16 & 0,10 & 0,11 \\
\hline $\begin{array}{c}\text { Edificação com muitas superfícies livres: em média 30\% de } \\
\text { áreas impermeáveis. }\end{array}$ & 0,21 & 0,22 & 0,14 & 0,16 & 0,09 & 0,10 \\
\hline $\begin{array}{l}\text { Zonas industriais e comerciais: em média } 72 \% \text { de áreas } \\
\text { impermeáveis. }\end{array}$ & 0,22 & 0,23 & 0,16 & 0,17 & 0,11 & 0,11 \\
\hline Campo e áreas verdes. & 0,18 & 0,20 & 0,12 & 0,14 & 0,09 & 0,10 \\
\hline Zonas florestais e vegetação densa. & 0,19 & 0,20 & 0,12 & 0,14 & 0,09 & 0,09 \\
\hline
\end{tabular}

Dos valores de "Cr" mapeados foram extraídas as suas áreas de abrangência na bacia hidrográfica do rio Atuba (Tabela 6) e destas realizada a sua média ponderada, ou seja, foram calculados os valores de " $\mathrm{Cr}_{\mathrm{m}}$ " para os anos de 1962, 1980, 2000 e 2020. 
NUNES, F. G.; FIORI, A. P. Análise da impermeabilização dos solos e aumento dos picos de vazão...

TABELA 6 - RELAÇÃO ENTRE O COEFICIENTE "Cr" E O PERCENTUAL DE ÁREAS OCUPADAS NA BACIA HIDROGRÁFICA DO RIO ATUBA. RELATION BETWEEN THE COEFFICIENT RUNOFF "Cr" AND THE PERCENTAGE OF BUSY AREAS IN THE ATUBA RIVER HYDROGRAPHIC BASIN

\begin{tabular}{|c|c|c|c|c|}
\hline \multirow{2}{*}{$\begin{array}{c}\text { Valores de "Cr" para condição III } \\
\text { de umidade antecedente }\end{array}$} & \multicolumn{4}{|c|}{ Área de cada parcela distinta em $\mathrm{km}^{2}$ / Anos } \\
\hline & 1962 & 1980 & 2000 & 2020 \\
\hline 0,09 & 16,39 & 10,5 & 4,43 & 0,5 \\
\hline 0,10 & 18,43 & 15,5 & 6,75 & 0 \\
\hline 0,11 & 1,26 & 5,3 & 9,71 & 12,8 \\
\hline 0,12 & 18,91 & 15,36 & 19,91 & 25,5 \\
\hline 0,14 & 8,42 & 7,48 & 4,31 & 0,9 \\
\hline 0,16 & 2,05 & 6,51 & 9,28 & 12,0 \\
\hline 0,17 & 0,028 & 2,03 & 4,41 & 6,8 \\
\hline 0,18 & 22,03 & 18,81 & 12,41 & 3,5 \\
\hline 0,19 & 27,16 & 20,52 & 22,16 & 25,5 \\
\hline 0,20 & 8,35 & 7,91 & 5,58 & 1,9 \\
\hline 0,21 & 2,74 & 12,42 & 16,55 & 19,0 \\
\hline 0,22 & 1,62 & 3,51 & 5,37 & 6,8 \\
\hline 0,23 & 0 & 1,35 & 4,20 & 7,5 \\
\hline 0,24 & 0 & 0,18 & 2,37 & 4,5 \\
\hline Corpos d'água & 0,05 & 0,05 & 0,11 & 0,11 \\
\hline Valor de " $\mathrm{Cr}_{\mathrm{m}}$ " calculado & 0,150 & 0,160 & 0,170 & 0,175 \\
\hline
\end{tabular}

Em uma bacia hidrográfica é de se esperar que o coeficiente de escoamento superficial varie com o tempo de retorno ou com a magnitude da enchente. Com o aumento da intensidade da chuva as perdas d'água não continuam as mesmas, ocasionando assim, o aumento deste coeficiente.
Levando em consideração esta variação Tucci (1993), reproduz a tabela elaborada (Wright \& Maclaughin 1962) que utiliza um multiplicador para o ajuste dos valores do coeficiente de escoamento superficial, de acordo com o tempo de retorno da chuva (Tabela 7 ).

TABELA 7 - FATOR DE CORREÇÃO DE "C" (WRIGHT \& MACLAUGHIN, 1962). FACTOR OF CORRECTION OF RUNOFF "C" (WRIGHT \& MACLAUGHIN, 1962)

\begin{tabular}{|c|c|}
\hline Tempo de retorno (anos) & Fator de correção \\
\hline 2 a 5 & 1,00 \\
\hline 10 & 1,05 \\
\hline 25 & 1,10 \\
\hline 50 & 1,20 \\
\hline 100 & 1,25 \\
\hline
\end{tabular}

FONTE: adaptado de Tucci (1993).

Aos valores de $\mathrm{Cr}_{\mathrm{m}}$ dos três anos em análise e do cenário futuro, foram acrescentados os fatores de correção da tabela acima, em função dos tempos de retorno (Tr), utilizados para os cálculos da intensidade das chuvas, obtendo-se assim, os valores finais de $\mathrm{Cr}_{\mathrm{m}}$ utilizados na determinação das vazões de pico simuladas pela fórmula racional (Tabela 8). 
NUNES, F. G.; FIORI, A. P. Análise da impermeabilização dos solos e aumento dos picos de vazão...

TABELA 8 - VALORES DE $\mathrm{Cr}_{\mathrm{m}}$ EM FUNÇÃO DO TEMPO DE RETORNO E DA INTENSIDADE DA CHUVA. MEAN VALUES OF RUNOFF BASED UPON RETURN TIME AND RAIN INTENSITY

\begin{tabular}{|c|c|c|c|c|}
\hline Tr (anos) & $\mathbf{1 9 6 2}$ & $\mathbf{1 9 8 0}$ & $\mathbf{2 0 0 0}$ & $\mathbf{2 0 2 0}$ \\
\hline 2 a 5 & 0,150 & 0,160 & 0,170 & 0,175 \\
\hline 10 & 0,160 & 0,170 & 0,180 & 0,185 \\
\hline 25 & 0,165 & 0,175 & 0,190 & 0,195 \\
\hline 50 & 0,180 & 0,192 & 0,205 & 0,210 \\
\hline
\end{tabular}

\section{ESTIMATIVA DA VAZÃO DE PICO PELO MÉTODO RACIONAL}

Após a obtenção de todos os parâmetros envolvidos na fórmula do Método Racional, modificada e adaptada para o presente estudo, calculou-se as vazões de pico para as diferentes situações de chuvas intensas, determinadas em função do tempo de retorno e de sua duração.

A duração da chuva intensa, ou seja, da chuva crítica que irá ocasionar a maior vazão foi considerada igual ao tempo de concentração desta na bacia, calculado para os anos de 1962, 1980, 2000 e cenário futuro (Tabela 9).

TABELA 9 - SIMULAÇÃO DA VAZÃO DE PICO NA BACIA DO RIO ATUBA PELA FÓRMULA RACIONAL. SIMULATION OF THE PEAK DISCHARGE IN THE BASIN ATUBA FOR THE RATIONAL EQUATION

\begin{tabular}{|c|c|c|c|}
\hline$t_{c}$ em min & Tr em anos & Intensidade da chuva $(\mathrm{mm} / \mathrm{h})$ & Vazão de simulação em m³/s \\
\hline \multicolumn{4}{|l|}{1962} \\
\hline \multirow{4}{*}{199,73} & 5 & 24,55 & 130,35 \\
\hline & 10 & 27,40 & 155,18 \\
\hline & 25 & 31,70 & 185,15 \\
\hline & 50 & 35,40 & 225,55 \\
\hline \multicolumn{4}{|l|}{1980} \\
\hline \multirow{4}{*}{145,70} & 5 & 31,97 & 181,06 \\
\hline & 10 & 35,70 & 214,83 \\
\hline & 25 & 41,30 & 257,30 \\
\hline & 50 & 46,10 & 313,30 \\
\hline \multicolumn{4}{|l|}{2000} \\
\hline \multirow{4}{*}{119,68} & 5 & 37,40 & 225,05 \\
\hline & 10 & 41,75 & 266,01 \\
\hline & 25 & 48,28 & 324,70 \\
\hline & 50 & 53,90 & 391,12 \\
\hline \multicolumn{4}{|l|}{2020} \\
\hline \multirow{4}{*}{106,95} & 5 & 40,75 & 252,43 \\
\hline & 10 & 45,48 & 297,82 \\
\hline & 25 & 52,60 & 363,07 \\
\hline & 50 & 58,75 & 436,71 \\
\hline
\end{tabular}

Na Equação Racional (equação 1) as variáveis e constantes para a bacia do rio Atuba assumem os seguintes valores:

$\mathrm{Q}_{\mathrm{p}}=$ vazão de pico dada em $\mathrm{m}^{3} / \mathrm{s}$;

$\mathrm{Cr}_{\mathrm{m}}=$ coeficiente de escoamento superficial real médio da bacia, para cada ano analisado, extraído da Tabela 7: $\mathrm{i}_{\mathrm{m}}=$ intensidade máxima da chuva, sobre toda a área drenada, para os tempos de concentração e períodos de retorno analisados;

$A=$ área da bacia igual a $127,43 \mathrm{~km}^{2}$; 
NUNES, F. G.; FIORI, A. P. Análise da impermeabilização dos solos e aumento dos picos de vazão...

\section{RESULTADOS E DISCUSSÃO}

Analisando o gráfico da Figura 2, que mostra a relação entre as vazões de pico simuladas pela fórmula racional com os valores dos coeficientes de escoamento superficial, verifica-se que o impacto da densificação da urbanização através da impermeabilização do solo gerou um incremento progressivo nos valores destes dois índices simulados, apresentando os valores plotados, uma reta ascendente ao longo dos quatro cenários estudados.

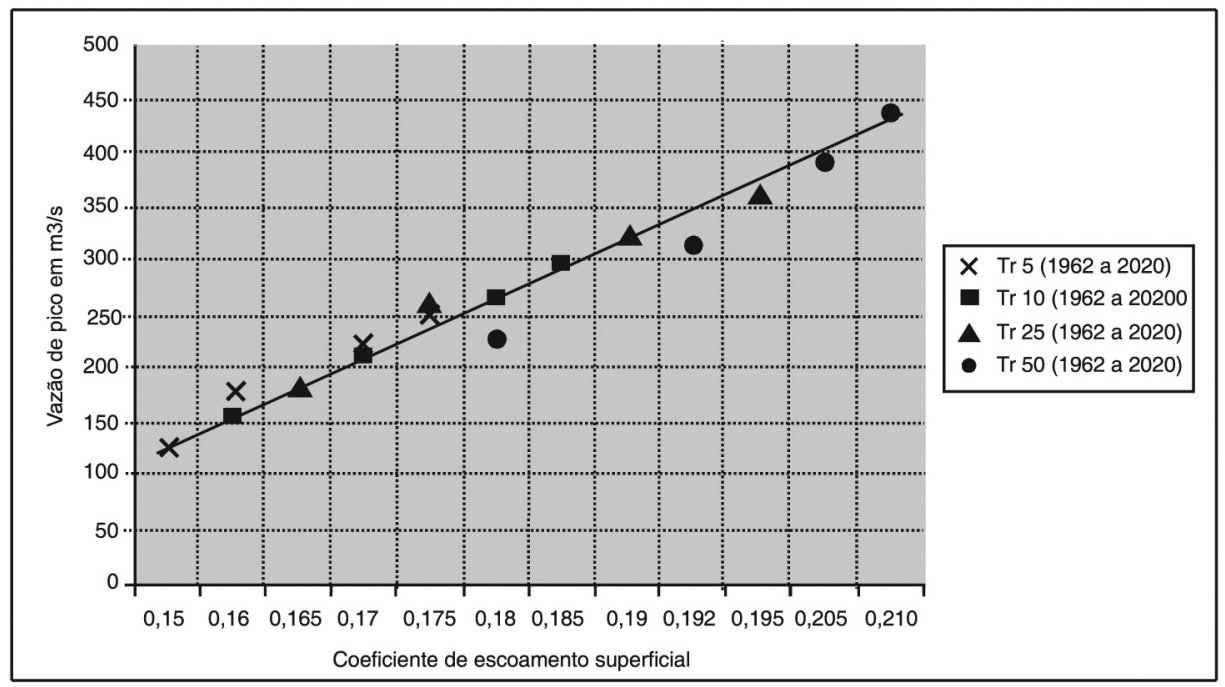

FIGURA 2 - COEFICIENTES DE ESCOAMENTO SUPERFICIAL X VAZÕES DE PICO SIMULADAS PARAABACIA DO RIO ATUBA. RUNOFF X SIMULATED OF PEAK DISCHARGE FOR THE BASIN ATUBA

Para uma chuva com Tr de 5 anos, o volume médio do coeficiente "C" passou de 0,150 para 0,170 , com um aumento de 13,33\% entre os anos de 1962 até 2000. Isto significa que o incremento do volume escoado na bacia, devido a uma impermeabilização de $30 \%$ do solo, proporcionou uma elevação de $72,85 \%$ da vazão de pico entre este dois períodos.

Do ano 2000 para uma projeção de um cenário de urbanização correspondente ao ano de 2020, ou seja, para a bacia em um estado de $45,5 \%$ de impermeabilização urbana, o coeficiente "C" passará de 0,170 para 0,175 com um aumento de $3 \%$, ocasionando uma vazão de pico $12,17 \%$ maior que a atual.

Para uma chuva com Tr de 10 anos, a percentagem de elevação do coeficiente " $C$ " de 0,160 para 0,180 foi igual a $12,5 \%$, gerando um aumento de $71,50 \%$ da vazão de pico entre os anos de 1962 a 2000. Na projeção do cenário futuro, em comparação com o atual, o acréscimo do valor de "C" de 0,180 para 0,185 será de $2,77 \%$, com uma vazão de pico $11,95 \%$ maior.

Seguindo a mesma tendência de evolução, para uma chuva com $\operatorname{Tr}$ de 25 anos, o coeficiente "C" de 0,165 para 0,190 cresceu $15,15 \%$ entre 1962 a 2000 , com um aumento de $75,43 \%$ da vazão de pico. Do ano de 2000 até o cenário futuro, a projeção do coeficiente "C" de 0,190 para 0,195 será igual a uma elevação de $2,63 \%$, acima do valor atual calculado, ocasionando um acréscimo de $11,82 \%$ da vazão.

Os valores do coeficiente "C" para $\operatorname{Tr}$ de 50 anos são os que ocupam os locais mais elevados do eixo das abscissas, no sentido das vazões de pico mais acentuadas, uma vez que o coeficiente de escoamento superficial tende a ser maior conforme aumentam os valores do tempo de recorrência.

No gráfico da Figura 2, observa-se ainda que o valor médio de "C" de 0,180 para 0,205 sofreu uma elevação de $14 \%$ entre 1962 a 2000, com uma vazão de pico $73,45 \%$ maior. Entre os anos de 2000 a 2020 o aumento do coeficiente "C" de 0,205 para 0,210 será de 2,44\%, o ocasionando um agravamento de $11,66 \%$ na vazão de pico simulada para um $\operatorname{Tr}$ de 50 anos. 
NUNES, F. G.; FIORI, A. P. Análise da impermeabilização dos solos e aumento dos picos de vazão...

\section{CONCLUSÕES}

Tendo-se por base os resultados da análise multitemporal da cobertura superficial dos solos, realizada através da quantificação das áreas permeáveis e impermeáveis, pode-se concluir que a bacia hidrográfica do rio Atuba sofreu um grande processo de impermeabilização urbana, no período compreendido entre anos 1962 a 2000. Em 1962 as áreas permeáveis que ocupavam $95,6 \%$ da bacia, reduziram-se para $85,3 \%$ em 1980 e para $70 \%$ em 2000 . Ao mesmo tempo, as áreas impermeáveis que ocupavam 4,5\% em 1962, aumentaram para $14,5 \%$ em 1980 e para $30 \%$ em 2000. Projetando-se este mesmo ritmo de adensamento urbano para um cenário futuro, ano de 2020 , tem-se a elevação das áreas impermeáveis para $45,5 \%$ de toda a área da bacia.

Através do emprego do Método Racional modificado, e adaptado para o presente estudo, verificou-se que a vazão de pico calculada para um tempo de retorno $(\mathrm{Tr})$ de 5 anos, que não ultrapassava 130,35 m³ $/ \mathrm{s}$ em 1962 , aumenta para $181,06 \mathrm{~m}^{3} / \mathrm{s}$ em 1980 e para $225,05 \mathrm{~m}^{3} / \mathrm{s}$ em 2000. Para o ano de 2020 este valor foi previsto para atingir $252,43 \mathrm{~m}^{3} / \mathrm{s}$.

Em um $\operatorname{Tr}$ de 10 anos, a vazão de pico aumentou de $155,18 \mathrm{~m}^{3} / \mathrm{s}$ em 1962, para 214,8 m³ $/ \mathrm{s}$ em 1980 e para $266,01 \mathrm{~m}^{3} / \mathrm{s} \mathrm{em} 2000$, sendo calculada para atingir $297,82 \mathrm{~m}^{3} / \mathrm{s}$ em 2020 .

No $\operatorname{Tr}$ de 25 anos, observa-se o mesmo incremento da vazão de pico. De 185,15m³/s em 1962 a vazão de pico agrava-se para $257,30 \mathrm{~m}^{3} / \mathrm{s}$ em $1980 \mathrm{e}$ para $324,70 \mathrm{~m}^{3} / \mathrm{s}$ em 2000 , com uma previsão de 363,07 $\mathrm{m}^{3} / \mathrm{s}$ para o ano de 2020. Para um Tr de 50 anos, a vazão de pico de $225,55 \mathrm{~m}^{3} / \mathrm{s}$ em 1962 eleva-se para 313,30 $\mathrm{m}^{3} / \mathrm{s}$ em 1980 e para $391,12 \mathrm{~m} 3 / \mathrm{s}$ em 2000 . No cenário futuro a vazão de pico será igual a $436,71 \mathrm{~m}^{3} / \mathrm{s}$.

Dos dados relacionado acima pode-se verificar que de um estado natural com apenas $4,5 \%$ de sua áreas impermeáveis em 1962, para um estado de $45,5 \%$ de área impermeável, atingida no ano de 2020 , a vazão de pico na bacia hidrográfica do rio Atuba praticamente duplicará, em todos os períodos de retorno analisados.

Analisando a variação do tempo de concentração da bacia do rio Atuba, em relação à impermeabilização dos solos ocorrida entre 1962 a 2000, pode-se verificar que para uma mudança na taxa de impermeabilização dos solos (Ai) de $4,5 \%$ para $30 \%$, o tempo de concentração da bacia passou de 3 horas e 35 min para 2 horas, ou seja, ocorreu um decréscimo no tempo de concentração de 1 hora e 30 min entre este período.

Para uma projeção futura (ano de 2020) com uma área de $45,5 \%$ de impermeabilização do solo, o tempo de concentração decairá para 1 hora e 45 min, sofrendo um decréscimo de 15 min entre o cenário atual para um cenário futuro.

A partir destas constatações pode-se concluir que a densificação urbana, com a consequente diminuição do tempo de concentração, vem aumentado o volume escoado e acelerando o percurso d'água desde o ponto mais distante da bacia até a seção de interesse. Este fator faz com que o rio Atuba, atinja cada vez mais rápido, o seu pico de vazão de cheias.

Os resultados obtidos no trabalho deixam bastante clara a correlação existente entre o aumento da impermeabilização do solo, aumento do coeficiente de escoamento superficial e aumento da vazão de pico. $\mathrm{Na}$ bacia hidrográfica do rio Atuba, em condição de solos próximos à saturação, foi verificado que com o acréscimo de $10 \%$ das áreas impermeáveis entre os anos de 1962 a 1980, ocorreu cerca de $6 \%$ de aumento no volume escoado, gerando um aumento de $38,5 \%$ na vazão de pico.

Para o período compreendido entre os anos de 1980 a 2000 verificou-se que o acréscimo de $15 \%$ das áreas impermeáveis, proporcionou também um aumento de cerca de $6 \%$ no volume escoado, gerando um aumento de $24 \%$ da vazão de pico. No prognóstico de um cenário futuro, com o incremento de $15,5 \%$ das áreas impermeáveis entre os anos de 2000 a 2020 ocorrerá um acréscimo de $3 \%$ do volume escoado, gerando um aumento de $12 \%$ na vazão de pico.

As técnicas de fotointerpretação digital e de Sistemas de Informação Geográfica utilizadas, apresentaram-se como uma boa alternativa para minimizar as dificuldades de espacialização dos principais elementos hidrológicos envolvidos no método empregado.

A partir destas técnicas pode-se distribuir os valores das variáveis hidrológicas ao longo de toda a bacia, conforme cada área homogenia, determinada pelos elementos físicos que as constituem, permitindo assim, que a contribuição de cada parcela seja contabilizada pela área que esta possui.

\section{AGRADECIMENTOS}

À Coordenação de Aperfeiçoamento de Pessoal de Nível Superior - CAPEs pelo apoio financeiro através da bolsa de doutorado, e ao Conselho Nacional de Desenvolvimento Científico e Tecnológico - CNPq pela bolsa de doutorado sanduíche no exterior. 
NUNES, F. G.; FIORI, A. P. Análise da impermeabilização dos solos e aumento dos picos de vazão...

\section{REFERÊNCIAS}

Chow, V. T. \& Maidment, D. R. \& Mays, L. W. 1988. Applied Hydrology. 1 ed. New York: McGraw Hill, 572 p.

EMBRAPA. Empresa Brasileira de Pesquisa Agropecuária \& IAPAR. Fundação Instituto Agronômico do Paraná. 1984. Carta de Solos do Levantamento e Reconhecimento dos Solos do Estado do Paraná. Londrina - PR, Convenio EMBRAPA/ SUDESUL/Governo do Estado do Paraná/IAPAR. Escala 1:600.000.

Fendrich, R. \& Obladen, N. L. \& Aisse, M. M. \& Garcias, C. M. 1997. Drenagem e Controle da Erosão Urbana. 1 ed. Curitiba: Champagnat, $194 \mathrm{p}$.

Fendrich, R. 2002. Coleta, Armazenamento, Utilização e Infiltração das Águas Pluviais na Drenagem Urbana. Tese (Doutorado - Pós Graduação em Geologia Ambiental) Universidade Federal do Paraná - UFPR, Curitiba - PR.

Genz, F. \& Tucci C. E. M. 1995. Infiltração em Superfícies Urbanas. Rev. Bras. de Engenharia - Associação Brasileira de Recursos Hídricos, Porto Alegre - RS, 13(1): 77-103.

Germano, A. \& Tucci, C. E. M. \& Silveira, A. L. L. 1998. Estimativa dos Parâmetros do Modelo IPH-II para Algumas Bacias Urbanas Brasileiras. Rev. Bras. de Recursos Hídricos - Associação Brasileira de Recursos Hídricos, Porto Alegre RS, 3(4): 103-120.
PROGRAMA SPRING 2005. INPE. Instituto Nacional de Pesquisas Espaciais, versão 4.2: Tutorial, São José dos Campos - SP, 1 CD-ROM.

IPH. Instituto de Pesquisas Hidráulicas - UFRGS; Faculdade de Engenharia Agrícola - UFPel. 2004. IP, H-S1: Manual de Fundamentos. Porto Alegre - RSVersão preliminar.

Paredes, E. A. 1994. Sistema de Informação Geográfica: Princípios e Aplicações de Geoprocessamento. 1 ed. São Paulo: Érica LTDA, 675 p.

Souza Pinto, N. L de \& Holtz, A. C. \& Gomide, F. L. S. 1976. Hidrologia Básica. 2 ed. São Paulo: Edgard Blücher, 278 p.

Tucci, C. E. M. 1993. Hidrologia: Ciência e Aplicação. 1 ed. Porto Alegre - RS, Editora da UFRGS, ABRH, EDUSP, v. 4, $942 \mathrm{p}$.

Tucci, C. E. M. \& Porto, R. L. \& Barros, M. T. 1995. Drenagem Urbana. 1 ed. Porto Alegre: Editora da UFRGS, 428 p. 